\title{
Biochemical parameters in patients suffering from head and neck cancer. Effect of nutritional support. Preliminary study
}

\author{
O. Casbarien ${ }^{1}$, P. Cresta ${ }^{1}$, C. Silva ${ }^{2}$, M. S. Feliu ${ }^{2}$, A. Badía ${ }^{1}$, N. López Delgado ${ }^{1}$, M. Perman ${ }^{3}$, \\ A. Navigante ${ }^{1}$ and N. Slobodianik ${ }^{2}$ \\ ${ }^{1}$ Angel Roffo Institute, ${ }^{2}$ Department of Nutrition. School of Pharmacy and Biochemistry, University of Buenos Aires and \\ ${ }^{3}$ Italian Hospital de Buenos Aires, Buenos Aires, Argentina
}

Nutritional status depression in persons suffering from head and neck cancer has been scarcely study. An inadequate nutrient intake could be the principal factor ${ }^{(1-3)}$. Previous study in a group of patients underwent conventional therapy showed changes in specific serum protein fractions, indicative of an impairment of nutritional status and inflammation ${ }^{(4)}$. The goal is the evaluation of nutritional status through the use of biochemical parameters in a subgroup of adult patients suffering from head and neck cancer before and after oral intake of a nutritional supplement.

The study was performed on seven patients $(n=7,45-60$ years old), who were attended in the Angel Roffo Institute, with local advanced head and neck cancer, underwent therapy with Chemoradiotherapy that completed oral intake of a nutritional supplement, during 60 days (Supportan ${ }^{\circledR}$, Fresenius-Kabi ). The supplement provides $500 \mathrm{Kcal} /$ day, with $27 \%$ of total calories cover by proteins, $40 \%$ by lipids ( $2 \mathrm{~g}$ EPA provided by fish oil, MCT and vegetal oils) and 33\% of carbohydrates. Patients had not severe mucositis - ulcera, bleeding and/or infection. Never of them had another severe toxicity by treatment nor had impairment of Perfomance Status. Blood samples were collected from fasting patients before (To) and after (Tf) the oral administration of the supplement. Specific serum protein fractions of potential usefulness in nutrition studies: transthyretin (TTR), transferrin (Transf), C3 (C3c), and C4 (C4c); acute phase serum fractions: ceruloplasmin $(\mathrm{Cp})$, haptoglobin $(\mathrm{Hp})$ and protein $\mathrm{C}$ reactive $(\mathrm{PCR})$ were measured by single radial immunodiffusion technique on agar gel layers (Biocientífica S.A., Argentina and Binding Site, UK). Biochemical parameters (Tf vs To) (mg/dl),expressed as X (sD) were: TTR: 21.1 (8.9) vs 22.9 (7.3); Transf: 188.9 (36.1) vs 207.3 (20.9); C3c:122.6 (24.9) vs 122.5 (52.3); C4c: 37.5 (7.4) vs 38.9 (11.1); Cp: 40.0 (6.7) vs 36.1(11.7), Hp: 334.0 (194.2) vs 245.6 (103.9); PCR: 1.4 (0.3) vs 1.6 (2.1). No differences between Tf and To were observed. These preliminary results suggest that the oral supplement administration was useful to maintain the initial biochemical nutritional profile. As a matter of fact, we consider a probable beneficial tendency in the implementation of this oral supplement to this type of treatment for head and neck carcinoma.

Supported by University of Buenos Aires (Code 20020100100044).

1. Ramos-Chaves M, Boléo-Tomé C, Monteiro-Grillo I, Camilo M \& Ravasco P. (2010) Oncologist. 15(5), 523-530.

2. Chasen MR \& Dippenaar AP (2008) Curr Oncol. 15(3), 117-122.

3. van Wayenburg CA, Rasmussen-Conrad EL, van den Berg MG et al. (2010) Health Care. 2(1), 16-21.

4. Feliu MS, Silva C, Cresta P et al. (2011) Proc. Nutr Soc (2012) 70 (OCE2), E33. 